\title{
Choice of Specimen Thickness in Axial Bright-Field STEM Tomography of Cells
}

\author{
Qianping $\mathrm{He}^{1}$, Richard D. Leapman ${ }^{1}$ \\ ${ }^{1 .}$ National Institute of Biomedical Imaging and Bioengineering, NIH, Bethesda, MD 20892
}

Axial bright-field electron tomography of biological specimens using scanning transmission electron microscopy (STEM) provides 3D reconstructions of cells from stained plastic sections that are around $1 \mu \mathrm{m}$ in thickness, which enables visualization of large cellular structures in their entirety [14]. This is achievable because in STEM there are no imaging lenses after the specimen so that chromatic aberration effects can be ignored in the presence of strong multiple inelastic scattering. Previously, we have shown that bright-field STEM tomography gives higher spatial resolution than dark-field STEM tomography for thick specimens [5, 6]. However, due to elastic scattering, the practical thickness range depends on the concentration of heavy atoms in the specimen, i.e., the fixation and staining protocols. Here, we describe a simple way to assess the usable thickness range.

The STEM signal $S_{B F}(\alpha)$ reaching a bright-field detector of semi-angle $\alpha$ (as indicated in Fig. 1A) depends on the specimen thickness $t$, the specimen tilt angle $\theta$, and the average mean free path for elastic scattering $\lambda_{e l}$ according to:

$$
S_{B F}(\alpha)=I_{0} \tau \exp \left[-t /\left(\lambda_{e l} \cos \theta\right)\right]
$$

Although the tomographic tilt series are collected in the STEM mode, the extent of elastic scattering can be assessed in the TEM mode, by inserting an objective aperture and measuring the transmitted beam intensity from a specimen region of interest using the microscope's CCD camera. The specimen is then moved out of the electron beam to measure the incident beam intensity.

As shown in Figs. 1B and IC, the usable thickness range for performing bright-field STEM tomography at a beam energy of $300 \mathrm{keV}$ from thick plastic sections of stained cells and tissues depends on the numbers of heavy atoms ( $\mathrm{Os}, \mathrm{U}, \mathrm{Pb}$, etc.) absorbed by the specimen, which is reflected by the average value of $\lambda_{e l}$. In the section of blood platelets in Fig. 1B, we find that it is possible to reconstruct specimens of thickness $\sim 1.4 \mu \mathrm{m}$, which is consistent with a measured value of $\lambda_{e l} \sim 500 \mathrm{~nm}$, and the equation above. However, for the retinal pigment epithelium sample in Fig. $1 \mathrm{C}$, it is not possible to obtain reconstructions of high quality from sections much thicker than $\sim 0.7$ $\mu \mathrm{m}$, which is consistent with a measured value of $\lambda_{e l} \sim 250 \mathrm{~nm}$ for this specimen.

Our results show that, whilst bright-field STEM tomography of thick sections is a powerful technique, it is critical to control the level of heavy atoms that are absorbed during specimen preparation. In this regard, it is straightforward to assess the heavy atom content by estimating the average elastic mean free path from TEM images prior to acquiring the STEM tomograms [7].

[1] M.F. Hohmann-Marriott et al, Nature Methods 6 (2009) p. 729.

[2] C.W. Graydon et al, J. Neurosci. 34 (2014) p. 8962.

[3] X. Chen et al, Proc. Natl. Acad. Sci. USA 112 (2015) p. E6983.

[4] I.D. Pokrovskaya et al, J. Thrombosis Haemostasis 13 (2015) p. 1.

[5] A.A. Sousa et al, Ultramicroscopy 109 (2009) p. 213. 
[6] A.A. Sousa et al, J. Struct. Biol. 174 (2011) p. 107.

[7] The authors thank Drs. B. Storrie and G. Zhang for providing the blood platelet specimen, and Drs. Q. Lu and C.J. Westlake for the providing the retinal pigment epithelium specimen. This research was supported by the intramural program of the National Institute of Biomedical Imaging and Bioengineering, NIH.
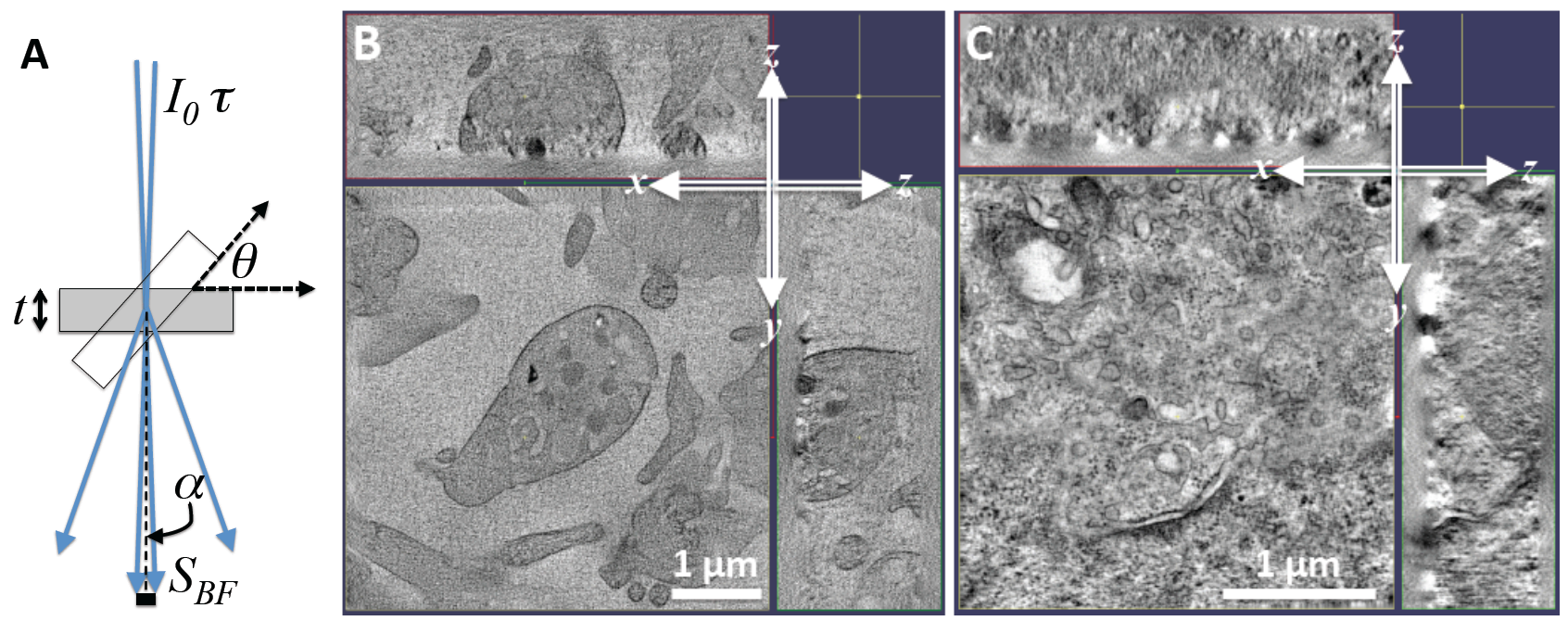

\begin{tabular}{|c|c|c|c|}
\hline \multicolumn{4}{|c|}{ Blood platelets } \\
$\left(I_{0} \tau=3,600\right.$ electrons/pixel) \\
\hline$t(\mu \mathrm{m})$ & $\theta$ & $t / \lambda_{e l} \cos \theta$ & $S_{B F}$ (electrons) \\
\hline 0.7 & $0^{\circ}$ & 1.4 & 888 \\
& $60^{\circ}$ & 2.8 & 219 \\
& $70^{\circ}$ & 4.1 & 60 \\
\hline \multirow{2}{*}{1.4} & $0^{\circ}$ & 2.8 & 219 \\
& $60^{\circ}$ & 5.6 & 13 \\
& $70^{\circ}$ & 8.2 & 1 \\
\hline
\end{tabular}

\begin{tabular}{|c|r|c|c|}
\hline \multicolumn{4}{|c|}{$\begin{array}{c}\text { Retinal pigment epithelium } \\
\left(I_{0} \tau=3,600 \text { electrons/pixel) }\right.\end{array}$} \\
\hline$t(\mu \mathrm{m})$ & $\theta$ & $t / \lambda_{e l} \cos \theta$ & $S_{B F}$ (electrons) \\
\hline 0.7 & $0^{\circ}$ & 2.8 & 219 \\
& $60^{\circ}$ & 5.6 & 13 \\
& $70^{\circ}$ & 8.2 & 1 \\
\hline \multirow{2}{*}{1.4} & $0^{\circ}$ & 5.6 & 13 \\
& $60^{\circ}$ & 11.2 & $<1$ \\
& $70^{\circ}$ & 16.4 & $<1$ \\
\hline
\end{tabular}

Figure 1. Effect of specimen thickness and tilt angle on detected signal in bright-field STEM tomograms of thick sections of stained cells, acquired at $300 \mathrm{keV}$ beam energy. (A) Specimen and detector geometry for section of thickness $t$ tilted to angle $\theta$, and detector semi-angle $\alpha$. For a probe current of $I_{0}$ electrons/s and a pixel dwell time $\tau$, the detected signal is $S_{B F}(\alpha)$. (B) $x-y, x-z$, and $y-z$ orthoslices through a relatively lightly stained section of human blood platelets with measured average elastic mean free path of $\sim 500 \mathrm{~nm}$, shows $3 \mathrm{D}$ reconstructions from a specimen of thickness $\sim 1.4 \mu \mathrm{m}$ (for $\sim 100 \mathrm{pA}$ probe current, $6 \mu \mathrm{s} /$ pixel dwell time (3,600 incident electrons $/$ pixel), $\pm 60^{\circ}$ tilt range (dual axes), and $10 \mathrm{mrad}$ detector semi-angle. Despite incident probe attenuation due to elastic scattering, as predicted from Eq. 1 and shown in the Table, there is sufficient signal at a $60^{\circ}$ tilt angle ( $\sim 13$ electrons/pixel) to reconstruct the blood platelets. (C) Orthoslices through a relatively heavily stained section of retinal pigment cells with measured average elastic mean free path of only $\sim 250 \mathrm{~nm}$, shows that it is not feasible to obtain 3D reconstructions from a specimen of thickness of $\sim 1.4 \mu \mathrm{m}$. The maximum thickness that can be analyzed is found to be approximately $0.7 \mu \mathrm{m}$, which is explained by the complete loss of signal predicted in the Table. 